\title{
A Metal-Only Reflectarray Antenna Using Double Split Square Ring Slot Element
}

\author{
Liu Meihua \\ Chongqing College of Electronic Engineering, Chongqing, 401331, China \\ 306110193@qq.com
}

\begin{abstract}
A novel metal-only reflectarray element with double split square ring slot laser cut into a metallic thick stainless steel plate is proposed in this paper. By using this unified slot structure, the dielectric substrate usually used in traditional reflectarray designs can be removed. The proposed technique can provide a more than $400^{\circ}$ phase range with relatively smoother slope. In order to verify the phase data, a $25^{\circ}$-beam-steering metalonly reflectarray operating at $12.5 \mathrm{GHz}$ is designed and simulated. A 1-dB gain bandwidth of $12 \%$ is achieved, which is slightly wider than that of reflectarray designs consisting of conventional patch elements. The proposed metal-only structure can better withstand the extreme outer space environment and effectively reduce the antenna cost.
\end{abstract}

Keywords: Metal-Only, Low Cost, Split Ring Slot, Reflectarray

\section{Introduction}

As an outstanding candidate for high-gain antennas, the microstip reflectarray antenna has received considerable attentions since its inception [1-2]. It combines some of the favorable features of reflectors and phased arrays, such as high radiation efficiency, low-profile, lightweight, and easy-to-deploy. Numerous techniques have been proposed to design the reflectarray elements, such as singlelayer single-resonant design [3-4], single-layer multi-resonant design [5-7], multilayer multi-resonant design [8-10], aperture coupled design [11-14], and so on. Among these techniques, the designed reflectarrays are usually supported by singlelayer or multiple-layer of grounded dielectric substrate. Even with the air substrate in some designs $[7,8,11]$, a thin dielectric layer is still required to keep the element in place. However, the operating environment in outer space is harsh, such as extremely high and low temperatures, which impose more stringent requirements on the design, so that a metal-only structure is commonly desired to withstand such environment.

Recently, some attempts have been made to innovating reflectarray designs without using any dielectric substrates. A rectangular groove element was proposed to realize 2D and 3D metal-only reflectarray [14 15], but the design was carved out of a metallic block with certain thickness which makes it relatively bulky. A difference patterns was realized by rotating the double split ring slot element cut into metallic plate [16]. A square slot element with stubs inside was proposed to realize a complementary reflectarray [17], and an 8\% 1-dB gain bandwidth was obtained by changing the stubs length, which is similar to bandwidth performance of reflectarray based on conventional patch element.

In this paper, a novel metal-only reflectarray element with the double split square ring slot laser cut into a metallic thick stainless steel plate is proposed. Numerical studies are carried out to understand the mechanism of the proposed element. In order to verify the obtained reflect phase data, a $25^{\circ}$-beam-steer metal-only reflectarray operating at $12.5 \mathrm{GHz}$ is designed and simulated. A 1-dB gain 
bandwidth of $12 \%$ is achieved with satisfactory radiation characteristics, which is slightly wider than that of reflectarray designs consisting of conventional patch elements.

\section{Element Design and Simulation}

The reflectarray concept is based on the scattering characteristics of elements. So the key technique in the design is how the individual elements are made to scatter the incident wave with the proper progressive phase shift necessary to produce a phase coherent beam in a specified direction. In reflectarray element designs, conventional microstrip elements, such as patches dipoles, and loops, have been popularly used to obtain proper reflection phase. By varying the element dimensions, a phase range close to $360^{\circ}$ is obtained in order to provide sufficient swing for phase compensations. These elements are isolated from each other, and a dielectric substrate is usually required to keep them in place. In order to remove the supporting dielectric substrate, the reflectarray elements need to be unified as a whole piece of metallic structure. Slot elements, a complementary geometry of these elements, become a natural choice for such design.

The top view and side view of the proposed double split square ring slots element is shown in Figure 1(a). An equivalent circuit model for this structure is depicted in Figure 1(b). This circuit model represents a double resonant structure. The capacitances $\mathrm{C} 1$ and $\mathrm{C} 2$ represent the capacitance of the outer and inner ring slot, and the inductances L1 and L2 represent the coupling between double ring slots in each element and the couple between unit element as formulated in [17].The phase of the reflected wave is controlled by changing the length 11 . Varying such sizes changes the impedance of the proposed element and therefore the phase of the reflected wave.

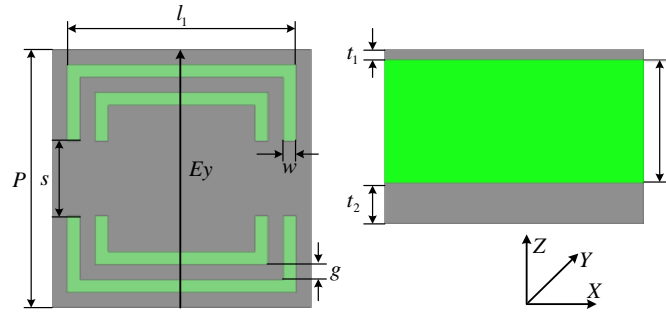

(a)

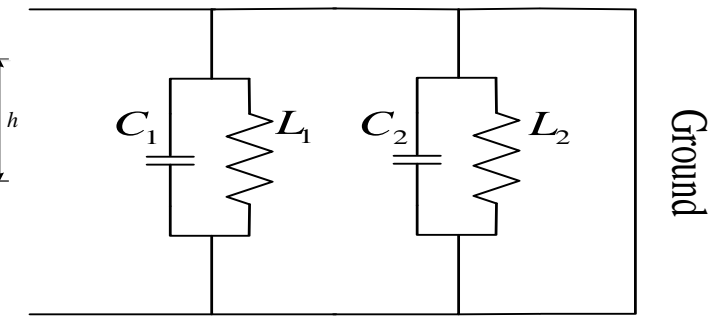

(b)

Figure 1. Configuration and Equivalent Circuit Model of the Proposed Element: (a) Top and Side View of the Configuration, (b) Equivalent Circuit Model

The reflected property of the proposed element is carried out by using an equivalent unit cell wave guide approach (WAG) with Ansoft High-Frequency Structure-Simulator (HFSS) software. A pair of perfect magnetic conductors in the $x$-direction and a pair of perfect electric conductors in the $y$-direction form the four waveguide side walls, which assumes that a uniform plane wave with a $y$-polarized electric field is normally incident on an infinite array of periodic structure. The reflected-phase is obtained by the phase of reflected coefficient S11. The double split square ring slots are cut into a $t_{1}=0.5 \mathrm{~mm}$-thick metallic steel-stainless plate with a period $P=12 \mathrm{~mm}$ that lean against a foam layer $\left(\varepsilon_{r}=1.067\right)$ of thickness $h=6 \mathrm{~mm}$, backed by a $t_{2}=1 \mathrm{~mm}$-thick steel plate that also acts as an antenna base. The optimized dimensions of the proposed element are given by: $w=0.6 \mathrm{~mm}, s=3.5 \mathrm{~mm}$, $g=0.7 \mathrm{~mm}$. With these dimensions, a more than $400^{\circ}$ phasing range with acceptable linearity and return loss can be obtained. It has been shown in the simulation that the reflected property of the proposed element is critically dependent on the split 
width and the slot width, but slightly dependent on the gap between two split slots. Therefore the split width and the slot width should be analyzed and optimized.

\subsection{The Effect of the Split Width}

To illustrate the effect of the split width, Figure 2 plots the reflected property of the proposed element with different split width at center frequency $12.5 \mathrm{GHz}$ while the slot width and gap between double slot are fixed at $w=0.6 \mathrm{~mm}$ and $g=0.7 \mathrm{~mm}$. In Figure 2 (a), one should note that the split width has a significant effect on the phasing range and the linearity of reflected phase curse. As the split width decreases, the phasing range increases, but a "knee" is observed in the phase curves, which results in worse linearity. It is because that decreasing the split width can make the two frequencies related to the dimensions of double split square ring slot closer to each other, which can be evidenced by the reflected amplitude curse shown in Figure 1(b). Note that the $s=3.5 \mathrm{~mm}$ curse has more than $400^{\circ}$ phasing range which is enough for reflectarray design, and shows better linearity which would result in a relatively broader bandwidth.

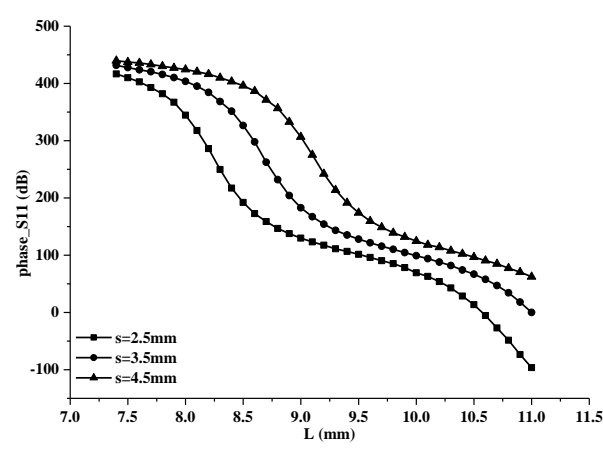

(a)

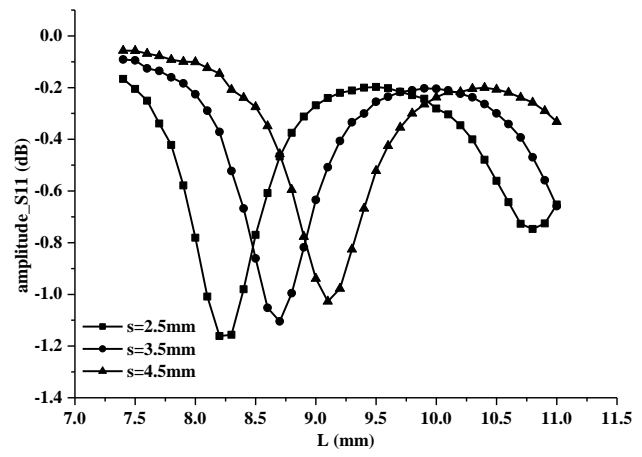

(b)

Figure2. The Reflected Property of the Proposed Element with Different Split Width: (a) Reflected Phase, (B) Reflected Amplitude

\subsection{The Effect of the Slot Width}

In order to explain the effect of the slot width, Figure 3 exhibits the reflected property of the proposed element with different slot width at center frequency $12.5 \mathrm{GHz}$ while the split width and gap between double slot are fixed at $s=3.5 \mathrm{~mm}$ and $g=0.7 \mathrm{~mm}$. From Figure $3(\mathrm{a})$, one should observe that the slot width also has an important influence on the phasing range and the linearity of reflected phase curse. As the slot width decreases, the variation tendencies of the reflected phase are almost the same as the situation that split width decreases, but the reflected amplitude in Figure 3(b) decreases noticeably, which would result in large return loss that is not expected in reflectarray design. Note that the $w=0.6 \mathrm{~mm}$ curse has not only enough phasing range but also acceptable return loss for reflectarray design. 


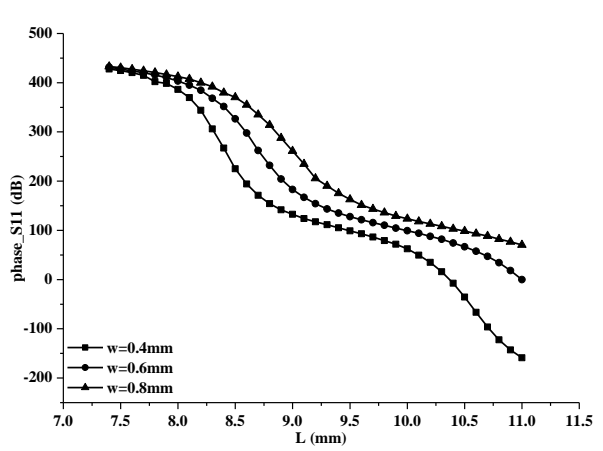

(a)

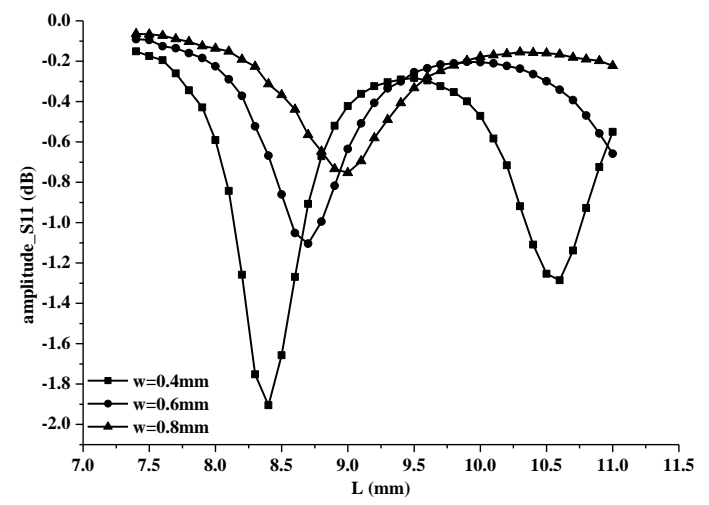

(b)

Figure 3. The Reflected Property of the Proposed Element with Different Ring Slot Width: (a) Reflected Phase, (b) Reflected Amplitude

\subsection{The Bandwidth Performance of the Proposed Element}

After parameters analysis, the bandwidth performance of the proposed element is exhibited. Figure 4 plots the reflected property of the proposed element over a frequency range of $11.5 \mathrm{GHz} \sim 13.5 \mathrm{GHz}$ centered. As shows in this figure, the relatively linear phase response and less than $1.1 \mathrm{~dB}$ return loss are achieved for this frequency range, which would result in a wide bandwidth and high gain reflectarray design.

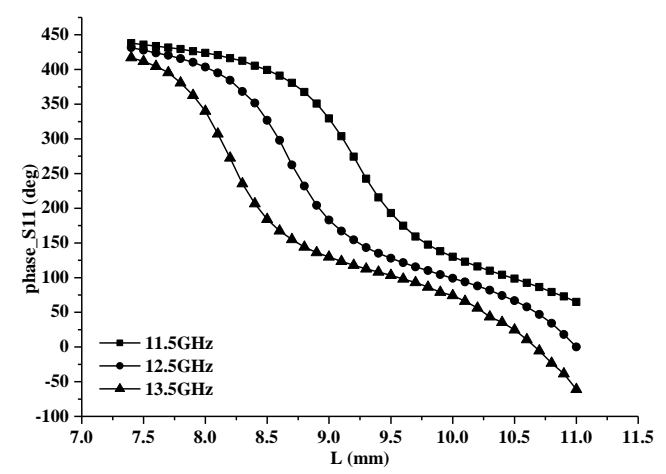

(a)

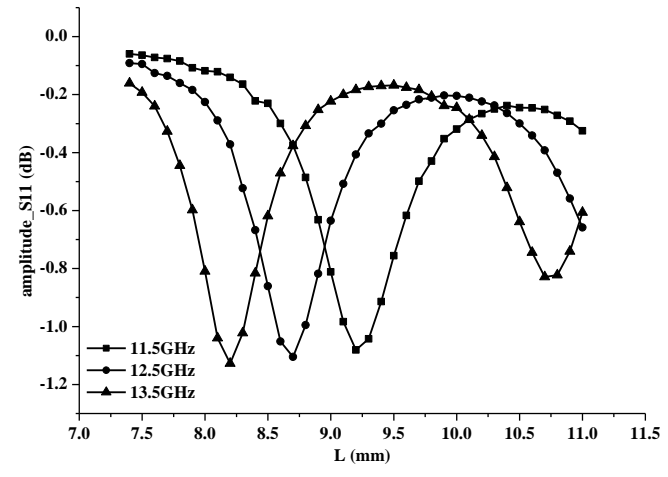

(b)

Figure 4. The Reflected Property of the Proposed Element with Different Frequencies: (a) Reflected Phase, (b) Reflected Amplitude

\section{Array Design and Simulation}

The main title (on the first page) should begin $13 / 16$ inches ( 7 picas) from the top edge of the page, centered, and in Times New Roman 14-point, boldface type. Capitalize the first letter of nouns, pronouns, verbs, adjectives, and adverbs; do not capitalize articles, coordinate conjunctions, or prepositions (unless the title begins with such a word). Please initially capitalize only the first word in other titles, including section titles and first, second, and third-order headings (for example, "Titles and headings" — as in these guidelines). Leave two blank lines after the title. 


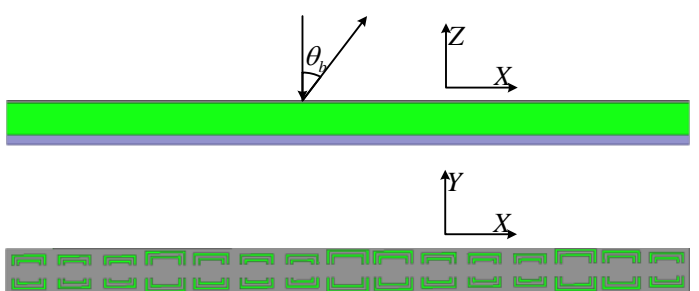

(a)

(b)

\section{Figure 5. Schematic Plan of Linear Array Excited by A Normal Incidence Y-Polarized Plane Wave: (a) Side View, (b) Top View}

In order to validate the reflected property of the proposed elements, a $25^{\circ}$-beamsteering linear reflectarray operating at $12.5 \mathrm{GHz}$ is designed. Figure 5 illustrates the basic geometry of this linear reflectarray with 15 proposed elements. In general, a feed horn should be placed at a facultative angle and distance from the reflectarray aperture. However, if the distance between the feed horn and reflectarray aperture is far enough, a plane wave with the same polarization as feed horn can be used to approximate the incident field. In this reflectarray design, the incident field is set as a y-polarization plane wave coming from the normal direction $\left(\theta=0^{\circ}\right)$. The required phase shift of the $n$th element to set the main beam off a given angle $\theta_{b}$ relative to broadside [2]:

$$
\phi_{n}=-k_{0} n P \sin \theta_{b}
$$

Then, the dimensions of the elements are chosen according to (1) and the reflected phase of the proposed element at $12.5 \mathrm{GHz}$. The Ansoft HFSS is used to simulate the design reflectarray model. The obtained radiation patterns are shown in Figure 6. The co-polarization patterns demonstrate that the main beam is pointing at $25^{\circ}$, quit agreeing with the design requirement. A higher side lobe around $\theta=0^{\circ}$ is observed in co-polarization patterns from Figure 6, which is caused by the specular reflection of metallic plate. The cr-polarization patterns are almost $-50 \mathrm{~dB}$ lower than the co-polarization, showing good polarization isolation.

In order to compare the gain bandwidth of the proposed element reflectarray and the conventional single-layer reflectarray, a same size reflectarray composed of square patches supported by $1.5 \mathrm{~mm}$-thick dielectric substrate with $\varepsilon_{\mathrm{r}}=2.65$ is designed and simulated. The normalized gain against frequency for the two reflectarrays is plots in Figure 7. As shown in Figure 7, a 1-dB gain bandwidth of $12 \%$ is achieved by the proposed element reflectarray, which is slightly wider than that for square patch reflectarray, namely $9 \%$.

\section{Conclusion}

A novel metal-only reflectarray element with the double split square ring slot laser cut into a metallic thick stainless steel plate is proposed. The unique unified slot structures adopted make sure that the dielectric substrate can be removed and that only metallic material is required in the reflectarray design. In order to understand the mechanism of the proposed element, the parameter analysis is carried out. Based on the obtained phase data, a $25^{\circ}$-beam-steer metal-only reflectarray operating at $12.5 \mathrm{GHz}$ is designed and simulated. A $1-\mathrm{dB}$ gain bandwidth of $12 \%$ is achieved, which is slightly wider than that of reflectarray designs consisting of conventional patch elements. The metal-only configuration proposed in this paper can better withstand extreme outer space environment, which makes it more suitable for space applications. Besides, the antenna cost is also remarkably reduced by removing the expensive microwave dielectric substrate, which makes it promising for commercial applications. 


\section{References}

[1] D. G. Berry, R. G. Malech and W. A. Kennedy, "The reflectarray antenna", IEEE Transactions of Antennas \& Propag, vol. 11, no. 6, (1963).

[2] J. Huang and J. A. Encinar, "Reflectarray Antennas", J. Wiley \& Sons, Hoboken, NJ, (2008).

[3] D. M. Pozar, S. D. Targonski and H. D. Syrigos, "Design of millimeter wave microstrip reflectarray", IEEE Trans. Antennas \& Propag, vol. 45, no. 2, (1997).

[4] D. M. Pozar and T. A. Metzler, "Analysis of a reflectarray antenna using microstrip patches of variable size”, Electro, Lett, vol. 29, no.8, (1993).

[5] M. R. Chaharmir, J. Shaker, M. Cuhaci and A. Ittipiboon, "Broadband reflectarray antenna with double square loops", Microw, Opt, Techn, Lett, vol. 48, no. 7, (2006).

[6] M. R. Chaharmir, J. Shaker, M. Cuhaci and A. Ittipiboon, "Broadband reflectarray antenna with double cross loops", Electro, Lett, vol. 42, no. 2, (2006).

[7] L. S. Ren, Y. C. Jiao, J. J. Zhao and G. Zhao, "A Novel Double-Petal Loop Element for Broadband Reflectarray", Prog, Electromagn, Res, Lett, vol. 13, (2010).

[8] J. A. Encinar, "Design of two-layer printed reflectarrays using patches of variable size", IEEE Trans, Antennas \& Propag, vol. 49, no. 10, (2001).

[9] J. A. Encinar and J. A. Zomaza, "Broadband design of three-layer printed reflectarrays", IEEE Trans. Antennas \& Propag, vol. 51, no. 7, (2003).

[10] N. Misran, R. Cahill and V. F. Fusco, "Design optimization of ring elements for broadband reflectarray antennas," IEE Proc, Microw, Antennas \& Propa, vol. 150, no. 6, (2003).

[11] A. W. Robinson, M. E. Bialkowski and H. J. Song, "An 137-element active reflect-array with dual-feed microstrip patch elements", Microw, Opt, Techn, Lett, vol. 26, no. 3, (2000).

[12] E. Carrasco, M. Barba and J. A. Encinar, "Aperture-coupled reflectarray element with wide range of phase delay", Electro. Lett, vol. 42, no. 12, (2006).

[13] S. Costanzo, F. Venneri and G. D. Massa, "Bandwidth enhancement of aperture-coupled reflect-arrays", Electro, Lett, vol. 42, no. 23, (2006).

[14] Y. H. Cho, W. J. Byun and M. S. Song, "Metallic-rectangular-grooves based 2D reflectarray antenna excited by an open-ended parallel-plate waveguide", IEEE Trans. Antennas \& Propag, vol. 58, no. 5, (2010).

[15] Y. H. Cho, W. J. Byun and M. S. Song, "High gain metal-only reflectarray antenna composed of multiple rectangular grooves", IEEE Trans, Antennas \& Propag, vol. 59, no. 12, (2011).

[16] D. Zelenchuk, and V. Fusco, "Double split ring slot FSS reflectarray for difference pattern generation", Electro, Lett, vol. 49, no. 1, (2013).

[17] W. X. An, S. H. Xu and F. Yang, "A Metal-Only Reflectarray Antenna Using Slot-Type Elements", IEEE Antenn, Wirel Prop, Lett, vol. 13, (2014).

[18] A. K. Rashid, B. Li and Z. X. Shen, "An Overview of Three-Dimensional Frequency-Selective Structures", IEEE Antennas and Propagation Magazine, vol. 56, no. 3, (2014). 